East African Medical Journal Vol. 86 No. 11 November 2009

HIV / AIDS AND PREGNANCY-RELATED DEATHS IN BLANTYRE, MALAWI

V.M. Lema, MBChB, MMed, Professor, Aga Khan University Nairobi, P.O. Box 25594-00603, Nairobi, Kenya, C. Kanyighe, MBBS, Registrar, J. Changole, SRN, SRM, Dip. Nursing, Research Nurse and E.V. Malunga, Dip. Bus. Admin., Executive Officer - Medical Audit, Department of Obstetrics and Gynaecology, University of Malawi, Blantyre, Malawi

Request for reprints to: Prof. V. M. Lema, Aga Khan University Nairobi, P.O. Box 25594 - 00603 , Nairobi, Kenya

\title{
HIV/AIDS AND PREGNANCY-RELATED DEATHS IN BLANTYRE, MALAWI
}

\author{
V.M. LEMA, C. KANYIGHE, J. CHANGOLE and E.V. MALUNGA
}

\begin{abstract}
Background: HIV/AIDS epidemic is one of the major factors affecting women's health and impeding national efforts to improve it especially in sub-Saharan Africa. Current evidence indicates that HIV/ AIDS is increasingly becoming a major cause or contributing factor to pregnancy-related deaths, almost overtaking the "traditional" causes and factors.

Objectives: To survey the contribution of HIV infection and AIDS to pregnancy-related deaths in Blantyre, Malawi.

Design: A retrospective, descriptive, facility-based survey.

Setting: The Queen Elizabeth Central Teaching Hospital, Blantyre, Malawi.

Subjects: All women recorded to have had pregnancy-related deaths between January 1, 1999 and December 31, 2000.

Results: There were 204 maternal deaths, 154 (75.5\%) direct, the top five causes being puerperal sepsis $(39.0 \%)$, postabortion complications $(31.2 \%)$, obstetric haemorrhage $(14.3 \%)$, eclampsia $(8.4 \%)$ and ectopic pregnancy $(3.9 \%)$. At least $38(18.6 \%)$ of the total were HIV positive or had AIDS. The main causes of deaths amongst these were meningitis $(23.7 \%)$, pneumonia $(18.4 \%)$, puerperal sepsis $(13.2 \%)$, postabortal sepsis $(10.5 \%)$, severe anaemia $(10.5 \%)$ and pulmonary tuberculosis $(10.5 \%)$ Of those who died of puerperal and postabortal sepsis in the whole study group $8.3 \%$ had HIV/AIDS. Of the in direct maternal deaths, $50(58 \%)$ were HIV positive or had AIDS.

Conclusion and recommendations: HIV/AIDS contributes to both direct and indirect maternal deaths in Malawi. National strategies to realise MDG5 targets should include addressing the HIV/AIDS epidemic within the entire population as well as scaling up contraceptive uptake and utilisation, especially amongst the most vulnerable groups.
\end{abstract}

\section{INTRODUCTION}

Maternal health has been on the international agenda for over two decades now. It is acknowledged that maternal well-being is critical for national social and economic development. Cognisant of that many countries have endorsed reduction of maternal deaths and by inference morbidities as a key developmental goal. Reduction of maternal deaths is also included in consensus emanating from various international conferences over the last twenty or so years (1-4). Majority of countries have initiated programmes in response thereto, with the support of international or regional agencies. While some countries have registered significant progress in reducing their national maternal mortality ratios, others have witnessed unprecedented increases (5-8), a fact which has raised serious concerns. These increases have been attributed to among other factors HIV infection, poor socio-economic status, civil strife and weak health systems (5,9-12).

Malawi's estimated national maternal mortality ratio (MMR) increased from 620/100,000 live births in 1992 to $1120 / 100,000$ live births $(13,14)$. The WHO/UNICEF UNFPA 2000 estimates on MMR (2004) gave a much higher figure of $1800 / 100,000$ live-births (5). Although institutional data on MMR is not necessarily representative of what pertains in the larger society especially in developing countries (5) studies conducted within health facilities in Malawi since 1989 have shown similar trends. Wiebenga (15), reported a MMR of 529/100,000 live births at the Queen Elizabeth Central hospital for 1989-1990. This figure decreased slightly between 1992 and 1994 to $476.5 / 100,000$ live births (16), but increased to $1041.8 / 100,000$ live births in 1999-2000 (17). 
Comparison among earlier and recent studies has also shown changes in the pattern of causes and predisposing factors thereto. Studies conducted between 1987 and 1994 showed that the leading causes were obstetric haemorrhage, puerperal sepsis, obstructed labour/ruptured uterus, complications of abortion and eclampsia in a descending order (15-19). Poor quality, lack of access to and delays in accessing and being offered appropriate emergency obstetric care were cited as major predisposing factors $(7,15-19)$. Recent studies in Malawi as well as other countries in the region have shown that puerperal and postabortal sepsis account for a very sizeable proportion of maternal deaths, with puerperal sepsis being the leading cause among the direct maternal deaths $(17,20,21)$. While the same predisposing factors are still operational in majority of cases, there is a growing body of evidence to suggest that HIV / AIDS is increasingly becoming a major contributing factor $(17,20,22-24)$.

Malawi has one of the highest HIV-1 seroprevalence rates globally, estimated at $14.1 \%$ $(6.9-21.4 \%)$ among the general population by UNAIDS, making it the eight most affected country in sub-Saharan Africa (25). The seroprevalence among antenatal mothers is also one of the highest globally and although it has been dropping lately, it was reported to be $25.6 \%$ in the Southern region of Malawi in 1996 (26). HIV / AIDS is responsible for the recent increase in mortality in the general population. Amongstyoung women aged 15-49 years HIV/ AIDS is reported to have led to an increase in mortality from 6.5 to 11.3 per 1000, between 1992 and 2000 (14). Young women aged 15-29 years are most vulnerable to HIV infection in SSA $(14,25)$. They are also the most fertile. An HIV infected woman is four times more likely to die than her uninfected colleague $(11,12)$.

Following the publication of the $\mathrm{WHO} /$ UNICEF/ UNFPA2000 estimates on MMR in 2004 and Malawi DHS (2004) results, there has been a renewed interest and concern regarding maternal mortality, its causes as well as predisposing factors in Malawi, with several calls for more indepth studies to identify the operational factors and possible interventions.

It was against this background that this study was designed and conducted. The aim was to identify the contribution of HIV / AIDS to pregnancy-related deaths in Blantyre, Malawi and possible strategies to facilitate the country's progress towards attainment of MDG5 targets.

\section{MATERIALS AND METHODS}

This study was conducted at the Queen Elizabeth Central, Hospital in Blantyre, Malawi. This is the largest public hospital nationally. It is the University
Teaching Hospital for medical doctors, nurses as well as various cadres of paramedics. The hospital serves as the district hospital for Blantyre District, a referral hospital for the Southern region of Malawi and neighbouring districts of the Central region. It also receives patients who are mainly self-referred with emergency obstetric conditions from areas of Mozambique bordering Southern Malawi.

The department of obstetrics and gynaecology handles an average of 30 deliveries per day, about $14.0 \%$ of which are through Caesarean section and 2500-3000 abortion-admissions per year $(16,17,27)$. Since 1992, the department has been holding maternal death audit meetings twice monthly, during which every maternal death occurring in the department is discussed thoroughly by a team of doctors and nurses / midwives, identifying the causes, predisposing factors and any other related factors. Where relevant, feedback is given to the referring health facility and the District Health Officer-Blantyre and the City's Medical Officer of Health for appropriate action.

This was a retrospective, descriptive study involving all women who suffered a maternal death, in the gynaecological, labour, antenatal and postnatal wards, operating theatres and intensive care unit, from January 1, 1999 to December 31,2000, both days inclusive. Case records of the mothers who died and reports from the above meetings were critically reviewed by the authors. The aim was to identify the social, demographic and reproductive characteristics of the women who died, the immediate causes of death and possible operational factors. The relevant laboratory tests and their results whenever available were also reviewed and documented.

Specially designed data transfer forms (DTFs) were used to collect the required information. Answers to the open-ended questions were coded and all the data subsequently analysed using the EPI-INFO 6.0 data analysis software. The numbers of deliveries and live born babies were obtained from the labour ward register.

\section{RESULTS}

There were 204 recorded maternal deaths and 19,581 live births during the two years under study. The annual numbers of deaths were more-or-less equal in each of the two years, 103 in 1999 and 101 in 2000.

Age distribution: The ages ranged from $16-40$ years. The modal age group was 20 to 24 years followed by 25 to 29 years. Adolescents (10 to 19 year olds) comprised $20.6 \%$ or one fifth of the total study group, and the third largest age group. More than half, 115 (56.4\%) of the mothers were youths, i.e. 15 to 24 years old. Those aged 35 years and above, made up only $8.8 \%$ of the total study group (Figure 1). 
Figure 1

Percentage distribution of the mothers by their age-groups

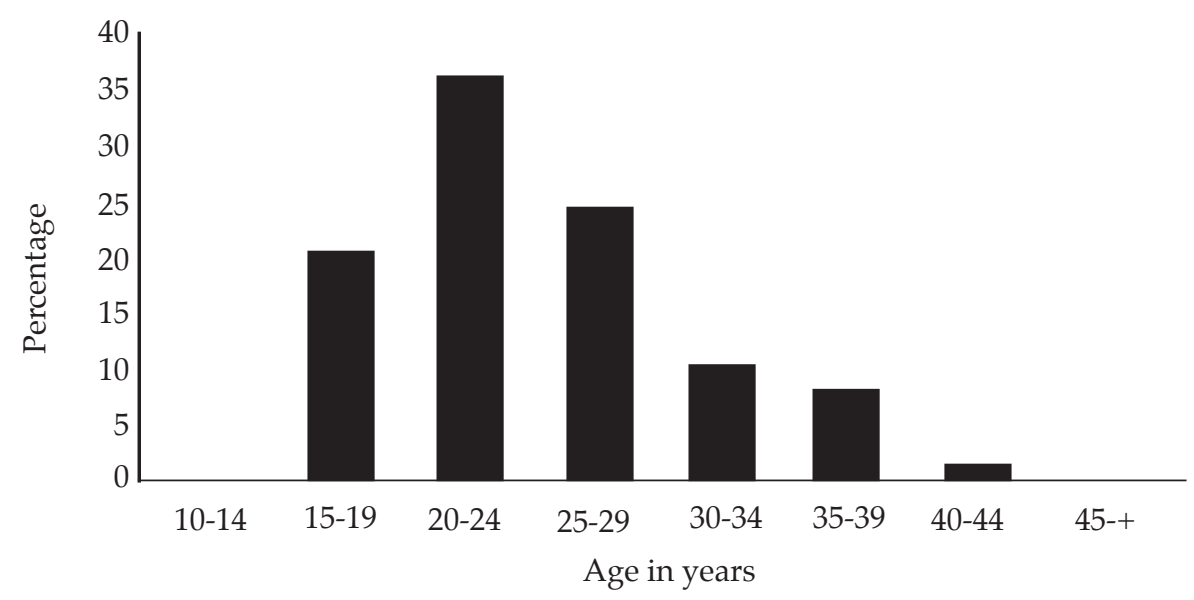

Figure 2

Percentage distribution of the mothers by their parities

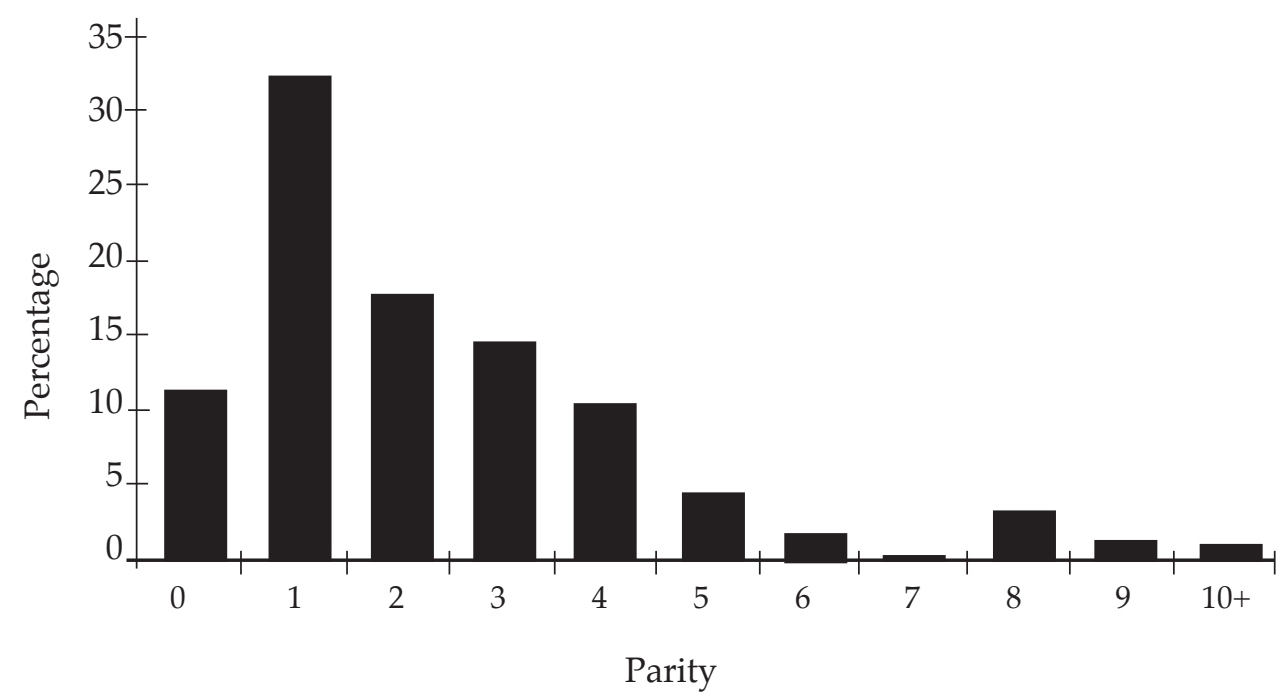

Parity: About two thirds of the mothers who died $(61.8 \%)$ were para 2 and less, i.e. of low parity. Of these, $11.3 \%$ were para 0 , i.e. this was their first pregnancy. The grandmultiparae (para 5 and above) comprised only $12.8 \%$ (Figure 2). There was a strong relationship between the individual's age and parity. The older one was the higher was the parity, as would be expected. Primiparae formed the largest single category, $(32.4 \%)$.

Causes of death: The main immediate causes of all the deaths were puerperal sepsis (29.4\%); post abortion complications (23.5\%); other infectious conditions $(20.1 \%)$; obstetric haemorrhage $(10.8 \%)$; eclampsia (6.4\%); and ectopic gestation (2.9\%) (Table 1).
Of the 204 maternal deaths, 154 (75.5\%) were due to direct causes, the main ones being puerperal sepsis $(39.0 \%)$, post abortion complications (sepsis and haemorrhage) $(31.2 \%)$, obstetric haemorrhage $(14.3 \%)$, eclampsia $(8.4 \%)$, ruptured ectopic gestation (3.9\%) and obstructed labour/ ruptured uterus (3.2\%) (Figure 3).

Sepsis was the main cause of death $(77.1 \%)$ among those with postabortion complications $(n=48)$. Puerperal and postabortal sepsis together accounted for nearly half, $97(47.5 \%)$ of all and two-thirds $(63.0 \%)$ of direct maternal deaths. Obstetric and postabortal haemorrhage on the other hand, was responsible for $16.2 \%$ of all deaths and $21.4 \%$ of direct maternal deaths. 
Table 1

Percent distribution of all patients by their immediate causes of death

\begin{tabular}{lrc}
\hline Cause of death & No. & $(\%)$ \\
\hline Puerperal sepsis & 60 & 29.4 \\
Postabortal complications & 48 & 23.5 \\
Other infections & 41 & 20.1 \\
Obstetric haemorrhage & 22 & 10.8 \\
Eclampsia & 13 & 6.4 \\
Ectopic pregnancy & 6 & 2.9 \\
Ruptured uterus/Obstructed labour & 5 & 2.5 \\
Heart failure & 4 & 2.0 \\
Not established & 5 & 2.5 \\
\hline Total & 204 & 100 \\
\hline
\end{tabular}

Figure 3

Direct causes of maternal deaths

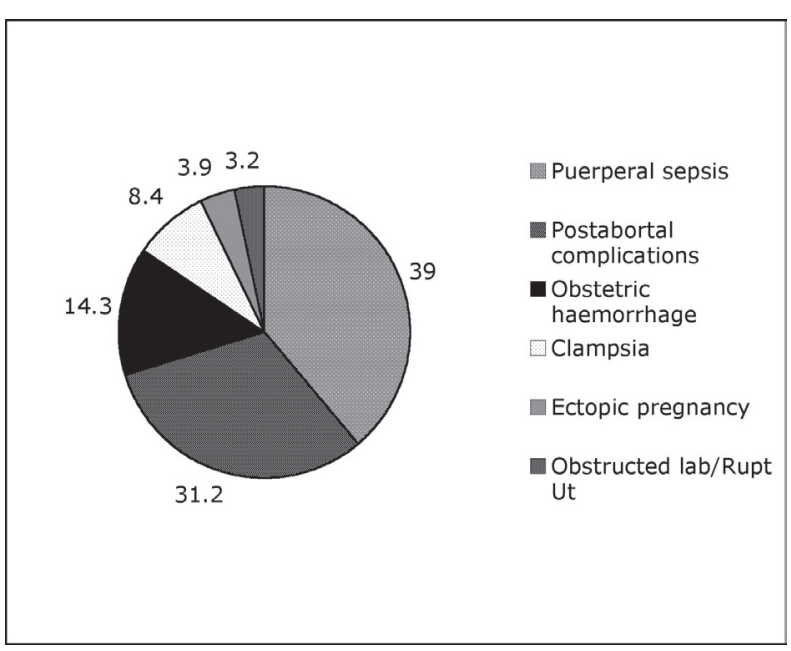

Fifty $(24.5 \%)$ were indirect maternal deaths, with infection, accounting for $82.0 \%$ of them. These included meningitis (bacterial and cryptococcal) (41.3\%), pneumonia (typical and atypical) $(36.3 \%)$, malaria $(7.5 \%)$, pulmonary tuberculosis $(7.5 \%)$ and severe gastroenteritis (7.5\%). Majority, 29 (58.0\%), of the indirect matemal deaths had HIV infection or AIDS.

Thirty eight $(18.6 \%)$ of all the mothers who died were HIV positive or had AIDS. The main causes of death were meningitis (23.7\%), pneumonia $(18.4 \%)$, puerperal sepsis $(13.2 \%)$, postabortal sepsis $(10.5 \%)$ and severe anaemia leading to congestive cardiac failure $(10.5 \%)$ and pulmonary tuberculosis (10.5\%). Disseminated Kaposi's sarcoma and malaria accounted for $5.3 \%$ and $2.6 \%$ respectively (Table 2 ).
Table 2

Percent distribution of the mothers with HIV/AIDS and their immediate causes of death

\begin{tabular}{lrc}
\hline Cause of death & No. & $(\%)$ \\
\hline Meningitis & 9 & 23.7 \\
Pneumonia & 7 & 18.4 \\
Puerperal sepsis & 5 & 13.2 \\
Postabortal sepsis & 4 & 10.5 \\
Severe anaemia and cardiac failure & 4 & 10.5 \\
Pulmonary tuberculosis & 4 & 10.5 \\
Gastroenteritis & 2 & 5.3 \\
Kaposi's sarcoma & 2 & 5.3 \\
Malaria & 1 & 2.6 \\
\hline Total & 38 & 100 \\
\hline
\end{tabular}

Five $(8.3 \%)$ of the mothers who died due of puerperal sepsis and four $(8.3 \%)$ of the ones who died of postabortal sepsis were HIV positive.

Puerperal and postabortal sepsis accounted for $61.8 \%$ of the deaths amongst adolescents. HIV / AIDS was responsible for at least $16.7 \%$ of the deaths amongst them, with the main immediate causes being meningitis, followed by pneumonia (Table 3). Adolescents comprised $18.4 \%$ of all the patients who had HIV / AIDS in this survey. Majority, (63.2\%) of the mothers who died of HIV infection related condition or AIDS were aged 20-29 years.

Table 3

Percent distribution of the adolescents by their immediate causes of death

\begin{tabular}{lrr}
\hline Cause of death & No. & $(\%)$ \\
\hline Postabortal sepsis & 15 & 35.7 \\
Puerperal sepsis & 11 & 21.2 \\
Postabortal haemorrhage & 4 & 9.5 \\
Ectopic pregnancy & 3 & 7.1 \\
Meningitis & 4 & 9.5 \\
Pneumonia & 4 & 9.5 \\
Eclampsia & 1 & 2.4 \\
\hline Total & 42 & 100 \\
\hline
\end{tabular}

\section{DISCUSSION}

Maternal death was chosen during the Millennium Declaration as the outcome with which progress towards attainment of MDG5 i.e. "to improve 
maternal health" will bejudged (4). Currentevidence indicates that some countries have made progress in reducing their maternal mortality ratios (5-8), while others have not made progress or their maternal mortality ratios have increased. Malawi is one of the countries which have witnessed an increase in the maternal mortality ratios between the early 1900 's and early 2000's $(5,13,14)$. The increases in maternal mortality ratios in sub-Saharan Africa have been attributed to the HIV / AIDS pandemic, poor socio-economic conditions, weak health systems and civil strife $(8,12,20,22)$. There is increasing evidence indicting that HIV / AIDS related maternal deaths are escalating considerably and changing the pattern of causes thereof. It is estimated that there are more than 2 million pregnancies among HIV infected women each year (22) and that most HIV infections in women are in resource-constrained settings where the risk of pregnancy-related death and morbidity is equally high $(5,8)$. Of the estimated 2.4-3.3 million HIV / AIDS-related deaths globally in 2005, about one-third occurred in sub-Saharan Africa (25).

Women are more susceptible to HIV-1 infection due to hormonal changes, vaginal microbial ecology and physiology and a higher prevalence of STl's. Women and especially young ones are up to five times more at risk of being infected than their male counterparts, and pregnant women are more likely to acquire HIV infection than their non-pregnant colleagues $(11,12,25,28)$. This fact is of great public health importance.

Malawi has both some of the highest HIV prevalence rates and maternal mortality ratios globally, $(5,25)$. The prevalence of STl's, especially genital ulcerative diseases is equally high (14). Some sexual practices which may increase HIV acquisition such as multiple partner relationships, dry sex are not uncommon in some segments of the population $(13,14)$.

HIV / AIDS was thought to have contributed to an almost doubling of the mortality among women aged 15-49 years from 6.5 to 11.3 / 1000 population between 1992 and 2000 in Malawi (14). This is also the reproductive segment of the population. The first indication of a possible contribution of HIV infection to maternal deaths in Malawi was documented by Wiebenga in 1992 (15) who showed that of the 151 deaths at the Queen Elizabeth Central Hospital in Blantyre in 1989-1990,20 (13.2\%) were HIV positive. The increase in both national and institutional maternal mortality ratios since then has coincided with the escalation of the HIV / AIDS epidemic and related deaths $(14,16)$. Muula et al $(23)$ stated that the increase in maternal mortality ratio between 1992 and 2000 in Malawi was associated with the HIV / AIDS epidemic, a fact supported by Bicego et al (24) and others $(22,23)$.
In our study, at least $18.6 \%$, almost one-fifth of the maternal deaths were HIV/AIDS related. Majority died of infections, which are also the major causes of deaths among HIV-infected individuals in sub-Saharan Africa $(9-12,17,20)$. A study in Uganda showed that HIV infected women were more likely to develop post-Caesarean section sepsis than those who were HIV-uninfected (29). The same could be said of women with normal delivery, especially due to poor quality of care in our health facilities and those with incomplete abortion, because of delay in seeking help as well as the quality of care offered. The actual contribution by HIV/AIDS to pregnancy-related deaths in Malawi is most likely much higher than reported in this study cognisant of the foregoing and the fact that not all women have HIV testing, deliver in a health facility, or that some may have HIV-related conditions and are admitted to the medical or surgical wards while pregnant or in the puerperal period and if they die they are often not documented as maternal deaths. Others may deliver normally, be discharged from hospital only to develop complications and die at home days later.

The increase in MMR in Malawi has also been associated with changes in the pattern of causes thereof. Not only have infections of any type become the major cause of pregnancy related deaths, but there has been an increase in the proportion of indirect maternal deaths $(15,16,17,20)$. Our findings and observations are supported by studies in other countries in the region. Ahmed et al (10) in Lusaka Zambia reported an increase in indirect maternal deaths $90 \%$ of whom died of HIV-related respiratory conditions. More-or-less similar observations have been made in other countries in the region $(9,11$, $12,28)$. HIV / AIDS is now acknowledged as a major cause or predisposing factor to maternal deaths in areas with high HIV prevalence. Sewankambo et al (11) showed that HIV positive women had a five time-risk of maternal death. Indirect maternal deaths increased from 23\% to 31\% (1998-2001) and those due to puerperal sepsis from $11 \%$ to $16 \%$ in South Africa mainly due to HIV/ AIDS (30).

On top of the health-related causes of deaths amongst these individuals, others may die due to neglect by family members or health care providers. In some situations health care providers may defer appropriate treatment, e.g. surgery, to anHIV-infected individual partly for fear of being infected.

Cognisant of the foregoing and national obligation to meet the indicators and targets for MDG5 as endorsed in 2000, it is not only critical that appropriate and reliable data on pregnancy-related deathsincluding their causes and predisposing factors are captured and analysed, but it is equally important that an attempt be made to analyse the direct and indirect maternal deaths separately (31). This is 
essential for programmatic purposes as it will not only facilitate identification of appropriate interventions for the respective causes and predisposing factors butfacilitate equitable allocation of resources as well. Maternal health needs to be looked at from the wider perspective of reproductive health.

\section{ACKNOWLEDGEMENT}

The authors are grateful to their colleagues in the department for their contributions during the maternal death audit meetings. We would wish to express our gratitude to the department and hospital administration for allowing us to use this data and publish the report.

\section{REFERENCES}

1. WHO - "Preventing the Tragedy of Matemal Death: Report of the Safe Motherhood Conference, Nairobi, Kenya, 1987" World Health Organisation, Geneva, Switzerland, 1987.

2. United Nations World Summit for Children (WSC), United Nations New York, 29-30 September 1990.

3. United Nations. Report on the International Conference on Population and Development. New York, United Nations, 1004.

4. United Nations (2000). Millennium Declaration. New York United Nations.

5. WHO/UNICEF/UNFPAMaternal Mortality in 2000 - Estimates developed by WHO, UNICEF, UNFPA. Geneva, WHO. 2004.

6. Senerviratne, H.R. and Rajapaksa, L.C. Safe motherhood in Sri Lanka: a 100-year march. Int. J. Gynaecol. Obstet. 2000; 70:113-124.

7. Campbell, O., Gipson, R., Issa, A.H., et al. National maternal mortality ratio in gypt halved between 1992-93 and 2000. Bull. World Health Org. 2005; 83: 462-472.

8. Abou Zahr, C., Wardlow, J., Stanton, C. and Hill, K. Maternal Mortality. World Health Stat. Quarterly. 1996; 49: 77 - 87.

9. Khan, M., Pillay, T., Moodley, J.M. and Connolly, C.A. Maternal mortality associated with tuberculosis-HIVI-co infection in Durban, South Africa. AIDS. 2001; 15:1857-1863.

10. Ahmed, Y., Mwaba, P., Chintu, C., et al. A Study of maternal mortality at the University Teaching Hospital, Lusaka, Zambia, the emergence of tuberculosis as a major non-obstetric cause of maternal deaths. Int. J. Tuberc. Lung Dis. 1999; 3: 657-680.

11. Ssewankambo, N.K., Gray, R.H., Ahmad, S., et al Mortality associated with HIV infection in rural Rakai District, Uganda. AIDS. 2000; 14: 2391-2400.

12. Le Coeur, S., Khlat, M., Halembokaka, G., et al. HIV and the magnitude of pregnancyrelated mortality in Pointe Noire, Congo. AIDS. 2005; 19: 69-75.
13. National Statistical Office. The Demographic and Health Survey - 1992. Zomba, Malawi. 1992.

14. National Statistical Office. The Demographic and Health Survey - 2000. Zomba, Malawi. 2000.

15 Wiebenga, J.E. Maternal mortality at Queen Elizabeth Central Hospital 1989-1990. Malawi Med. J. 1992; 8: 19-23.

16. Mtimavalye, L.A.R. Maternal deaths at the Queen Elizabeth Central Hospital-1992 -1994. Paper presented at the SASOG Annual Conference, Sun City, South Africa. August 1996.

17. Lema, V. M., Changole, J., Kanyighe, C. and Malunga, E. V. Maternal mortality at the Queen Elizabeth Central Teaching Hospital, Blantyre, Malawi. East Afr. Med. J. 2005; 82: 3-9.

18 Ministry of Health and population. Malawi Safe Motherhood Strategic Plan. Lilongwe. Malawi. 1995.

19 Family Care International. The Safe Motherhood Action Agenda: Priorities for the Next Decade. Report of the Safe Motherhood Technical Consultation. Colombo. Sri Lanka. October 18 - 23, 1987.

20. MalawiSafe Motherhood Program. Safe Motherhood - Helping to Prevent Maternal Deaths. Ministry of Health and population, Lilongwe. Malawi. 2000.

21. Ratsma, Y.E.C. “The Confidential Enquiries into Institutional Maternal Deaths in the Southem Region of Malawi _ 2001. Unpublished Report of the Safe Motherhood Project. Southern Region of Malawi.

22. Mcintyre, J. Maternal health and HIV. Reprod. Health Matters 2005; 13: 129-135.

23. Muula, A.S. and Phiri, A. Did Maternal Mortality ratio increase in malawi between 1992-1998? Review of demographic and health surveys and other data sources. Trop. Doc. 2003; 33: 182-185.

24. Bicego, G., Boerma, J.J. and Ronsman, C. The effect of AIDS on maternal mortality in Malawi and Zimbabwe AIDS. 2002; 16:1078-1081.

25. UNAIDS. HIV / AIDS estimates and data, 2005, 2006 report 0 the global AIDS epidemic. WHO, Geneva.

26. Verhoeff, F.H., Brabin, B.J., hart, C.A., et al. Increased prevalence of malaria in HIV-infected pregnant women and its implications for malaria control. Trop. Med. Int. Hlth. 1999; 4: 5-12.

27. Lema, V.M. and Thole, G.C. Incomplete abortion at the Teaching Hospital Blantyre Malawi. East Afr. Med. J. 1994; 71:727-735.

28. Gray, R.H., Li, X., Kigozi, G., et al. Increased risk of incident HIV during pregnancy in Rakai Uganda: a prospective study. Lancet. 2005; 366: 1182-1188.

29. Bjorklund, K., Mutyaba, T., Nabunya, E. and Mirembe, F. Incidence of post-caesarean section infection in relation to HIV status in a setting with limited resources. Acta Obstel. Gynaecol. Scand. 2005; 84: 927-928.

30. Moodley, J. and Pattinson, R.C. Maternal deaths in South Africa. S. Afr. Med. J. 2003; 93:354.

31. Stecklov, G. Maternal mortality estimation:Separating pregnancy-related and non-pregnancy-related risks. Stud. Fam. Plan. 1995; 26: 33-38, 